\title{
Capacidade de um sensor ótico em quantificar a resposta da cana-de-açúcar a doses de nitrogênio
}

\author{
José P. Molin ${ }^{1}$, Flávia R. Frasson ${ }^{2}$, Lucas R. Amaral ${ }^{3}$, Fabrício P. Povh ${ }^{3}$ \& José V. Salvi ${ }^{3}$
}

\begin{abstract}
RESUMO
Técnicas de agricultura de precisão são alternativas para otimizar a utilização de insumos, dentre eles o nitrogênio. 0 presente trabalho teve como objetivo verificar a possibilidade de uso de um sensor ótico ativo comercial na cultura da cana-de-açúcar, verificando sua capacidade em identificar a resposta da cultura a diferentes doses de nitrogênio (N ). Para tanto um experimento em blocos ao acaso foi instalado em área experimental de cana-de-açúcar de terceiro corte, colhida mecanicamente, com diferentes doses de $\mathrm{N}\left(0,50,100,150\right.$ e $\left.200 \mathrm{~kg} \mathrm{ha}^{-1}\right)$ e quatro repetições. Aos 30, 60 e 90 dias após o corte (DAC) realizou-se a mensuração do NDVI (índice de vegetação por diferença normalizada) a partir do sensor ótico. Não se obteve resposta do NDVI mensurado aos 30 e $60 \mathrm{DAC}$, porém aos $90 \mathrm{DAC}$ se verificou que o sensor foi capaz de diferenciar as doses de $\mathrm{N}$ aplicadas, correlacionando-se positivamente com a produtividade final de colmos obtida. Este trabalho dá subsídios a trabalhos de mesma natureza que visem a gestão da adubação nitrogenada em cana-de-açúcar a partir de sensores óticos.
\end{abstract}

Palavras-chave: agricultura de precisão, sensoriamento remoto, NDVI

\section{Capability of an optical sensor in verifying the sugarcane response to nitrogen rates}

\begin{abstract}
Techniques of precision agriculture are alternatives to optimize the use of inputs, among them nitrogen. The present work had as its objective to verify the possibility of use of a commercial optical sensor in sugarcane, verifying its capability in identification of the crop response to different nitrogen $(N)$ rates. An experiment was installed in randomized blocks in an experimental area with third sugarcane ratoon, harvested mechanically, with different $\mathrm{N}$ rates $\left(0,50,100,150\right.$ and $\left.200 \mathrm{~kg} \mathrm{ha}^{-1}\right)$ and four repetitions. At 30,60 and 90 days after the harvest (DAH) the measuring of NDVI (normalized difference vegetation index) was accomplished starting from the optical sensor. No response was verified of NDVI on measurements at the 30 and $60 \mathrm{DAH}$, however at $90 \mathrm{DAH}$ the sensor was capable to differentiate the doses of applied $N$, positively correlated with the sugarcane final production. This work gives subsidies to studies of same nature that seek the management of the nitrogen fertilization in sugarcane using optical sensor.
\end{abstract}

Key words: precision agriculture, remote sensing, NDVI

\footnotetext{
${ }^{1}$ ESALQ/U SP, Av. Pádua Dias, 11, CP 9, CEP 13418-900, Piracicaba, SP. Fone: (19) 34294165. E-mail: jpmolin@usp.br 2 Engenheira Agrônoma, Mestre em Máquinas Agrícolas. Fone: (18) 97252970. E-mail: flafrasson@yahoo.com.br

3 Pós-graduandos do PPG em Fitotecnia, ESALQ/U SP. Fone: (19) 34478509. E-mail: lucasamaral@agronomo.eng.br; fppovh@gmail.com; jvsalvi@yahoo.com.br
} 


\section{INTRODUÇÃO}

O setor canavieiro brasileiro experimenta uma fase de expressivo crescimento, impulsionado pelo aumento na demanda por etanol em substituição aos derivados de petróleo (Segato et al., 2006). Tal expansão requer a aplicação de processos mais eficientes para melhoria da produtividade e da qualidade dos produtos.

Sob esta ótica a adubação nitrogenada das lavouras de canade-açúcar deve merecer inovações. É uma das operações que mais demandam esforços de pesquisadores em todo o mundo, por se tratar de um elemento com dinâmica complexa no solo, sujeito a diversos mecanismos de perda e potencial causador de impactos ambientais. Desta forma, técnicas de sensoriamento remoto, aliadas às técnicas de agricultura de precisão, como a aplicação localizada de insumos em tempo real, de acordo com as necessidades específicas dentro de cada lavoura, podem refletir consideravelmente na melhoria do processo, com potencial retorno econômico.

Uma das técnicas de se avaliar indiretamente o estresse causado pela deficiência de nitrogênio nas culturas, é obtida pela mensuração da quantidade de clorofila nas folhas utilizando-se medidores de clorofila (Piekielek et al., 1995). Outras técnicas têm sido propostas para a determinação da demanda de nitrogênio pelas culturas, dentre elas o uso de imagens aéreas e orbitais, e o uso de sensores óticos terrestres. Wright et al. (2004), estudando essas técnicas em lavoura de trigo concluíram que mensurações de valores de refletância realizadas com sensores ótico terrestres, calculando-se o índice de vegetação por diferença normalizada (NDVI), se correlacionam melhor com parâmetros da cultura (produtividade, teor de $\mathrm{N}$ foliar e de proteína nos grãos) do que a obtenção dos mesmos índices a partir de imagens de satélite e fotografias aéreas. Referidos autores também apontaram que a aquisição de dados com sensores terrestres é menos dependente do clima, a coleta de dados pode ser feita aproveitando-se outras operações e os dados são disponibilizados em tempo real ou logo após sua coleta.

Sabe-se que a deficiência de $\mathrm{N}$ causa mudanças no comportamento espectral da radiação refletida pelas folhas das plantas, em virtude das alterações sofridas na falta do nutriente (Tarpley et al., 2000), sendo possível, através da mensuração dessas alterações, realizar a recomendação e aplicação da adubação nitrogenada de acordo com a real demanda das culturas, fato que já vem ocorrendo em milho (Blackmer et al., 1996; Teal et al., 2006), trigo (Stone et al., 1996; Raun et al., 2002; Wright et al., 2004; Povh et al., 2008; Grohs et al., 2009), algodão (Sui et al., 2005; Motomiya et al., 2009), cevada (Kim et al., 2005; Povh et al., 2008; Grohs et al., 2009) e citros (Min \& Lee, 2005), entre outras.

Sendo assim a disponibilização de sensores óticos ativos terrestres no mercado esta possibilitando avanços na pratica da adubação nitrogenada em varias culturas, com a perspectiva de se automatizar a decisão relativa a doses a serem aplicadas a partir de mensurações e intervenções em tempo real; entretanto ainda não se tem conhecimento suficiente sobre o comportamento espectral da cana-de-açúcar, sobretudo após o advento dos sensores óticos ativos que utilizam comprimentos de onda já consagrados em outras culturas. Em vista disto, o objetivo desta pesquisa foi, em uma investigação prospectiva, verificar a capacidade de um sensor ótico ativo comercial em identificar a resposta da cana-de-açúcar a diferentes doses de nitrogênio aplicadas.

\section{Material E MÉTODOS}

O experimento foi conduzido em área experimental de canade-açúcar de terceiro corte, na região de Assis - SP com coordenadas aproximadas de $22^{\circ} 40^{\prime} \mathrm{S}$ e $50^{\circ} 25^{\prime} \mathrm{O}$. O solo é classificado como Latossolo Vermelho Amarelo distrófico(LVA), em Ambiente de Produção classificado como “D” (Prado, 2005), por apresentar produtividade média dos cinco cortes entre 72 e $80 \mathrm{t} \mathrm{ha}^{-1}$, capacidade de agua disponível media-baixa e CTC media-alta.

Os tratamentos consistiram de quatro doses de nitrogênio (50,100,150 e $\left.200 \mathrm{~kg} \mathrm{ha}^{-1}\right)$, aplicados manualmente em cobertura, na forma de uréia, aos 23 dias após o corte (DAC), realizado em 26 de setembro de 2006, além de uma testemunha sem a aplicação de $\mathrm{N}$, com delineamento experimental em blocos ao acaso com quatro repetições.

A variedade utilizada foi a RB855536, com colheita mecânica do experimento em agosto de 2007, deixando-se sobre o solo os resíduos da colheita. Cada parcela contava com seis fileiras de plantas espaçadas $1,40 \mathrm{~m}$, com $15 \mathrm{~m}$ de comprimento. Os dados foram coletados nas quatro fileiras centrais descartandose uma de cada lado da parcela, como bordadura. Segundo UDOB (2007), a variedade escolhida apresenta alta produtividade agrícola e industrial em ambientes de produção B, C, D e E, porte médio, habito de crescimento ereto, ótima brotação, alto perfilhamento, bom fechamento entre as fileiras alto teor de sacarose e maturação média. Recomenda-se utilizar ambientes sem deficiência hídrica pronunciada no outono/ inverno e de boa fertilidade.

As leituras foram realizadas aos 30, 60 e 90 DAC, utilizandose o sensor otico ativo portátil GreenSeeker Red Hand Held ${ }^{\mathrm{TM}}$ (NTech Industries, Inc., Ukiah, CA) (Figura 1), o qual utiliza LEDs (light emitting diodes) que emitem luz em $660 \mathrm{~nm}( \pm 10$ $\mathrm{nm}$, vermelho) e $770 \mathrm{~nm}$ ( $\pm 15 \mathrm{~nm}$, infravermelho próximo), capta a refletância dos alvos por um fotodiodo detector e calcula automaticamente alguns índices de vegetação, sendo que neste trabalho foi utilizado o índice de vegetação por diferença normalizada (NDVI), com valores obtidos a partir da Eq. 1. O fotodiodo detector capta informações em uma frequência duas vezes maior que os emissores, estimando e anulando o efeito da refletância oriunda da luz solar e de outras fontes, o que possibilita seu uso em qualquer situação de luminosidade.

$$
\mathrm{NDVI}=\frac{(\rho \mathrm{IVP}-\rho \mathrm{V})}{(\rho \mathrm{IVP}+\rho \mathrm{V})}
$$

em que:

$\mathrm{IVP}$ - refletância no infravermelho próximo

$\rho \mathrm{V}$ - refletância no vermelho 


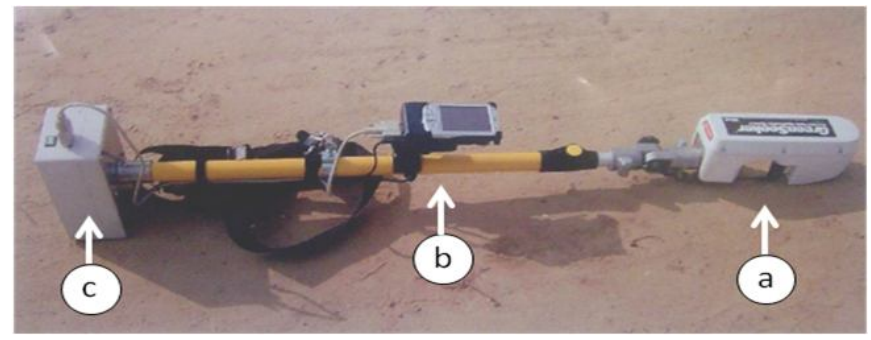

Figura 1. Sensor ótico ativo utilizado (G reenSeeker Hand Held ${ }^{T M}$ ). (a) Sensor, com emissor e receptor de luz; (b) PD A equipado com GPS para coleta de dados; (c) bateria portátil

O sensor foi utilizado manualmente, a uma altura média de 1,00 m de distância do dossel das plantas, com largura constante de mensuração de $0,61 \mathrm{~m}$, coletando até dez dados por segundo, de forma dinâmica de acordo com as especificações do fabricante, isto é, apenas uma fileira de plantas por vez. As avaliações aos 30, 60 e 90 DAC ocorreram quando a cultura apresentava altura média de 10, 25 e $45 \mathrm{~cm}$, respectivamente, medidas a partir da primeira folha com aurícula visível (altura de palmito). Em cada avaliação as leituras foram realizadas na área útil das parcelas, sendo a média dos valores de NDVI considerada o valor de NDVI representativo de cada parcela.

Para determinação da resposta da cultura dada pela produtividade final de colmos, realizou-se a colheita total da área útil das parcelas com pesagem em balança embarcada em caminhão.

Os dados foram submetidos a análise de variância, verificando-se a significância pelo teste F de Snedecor e, quando significativos, as médias foram comparadas pelo teste Tukey a 5\% de probabilidade, análise de regressão e correlação linear de Pearson.

\section{RESULTADOS E DISCUSSÃO}

Os valores médios entre os tratamentos aplicados à cultura e a resposta da variedade através do NDVI mensurado e seus respectivos coeficientes de variação $(\mathrm{CV})$, tal como a resposta na produtividade final de colmos são apresentadas na Tabela 1. Os valores do CV para o NDVI foram baixos, com valor médio máximo de 7,5\% aos 60 DAC e mínimo de 4,3\% aos 30 DAC, devido a uniformidade na brotação. Nota-se, entretanto, redução no CV aos 90 DAC (5,8\%), causada pelo aumento da biomassa, o que faz com que o valor do NDVI tenda a entrar na zona de saturação e, com isto, diminuir a variabilidade das leituras. Tal fato pode ser percebido quando se compara o comportamento do NDVI mensurado ao longo do experimento, apresentando tendência de médias crescentes, sendo de 0,221 aos 30 DAC e 0,626 aos 90 DAC.

$\mathrm{Na}$ análise estatística dos tratamentos (Tabela 1), constatouse que não houve efeito significativo dos tratamentos nos valores de NDVI mensurados aos 30 e 60 DAC devido, provavelmente, à baixa demanda inicial de $\mathrm{N}$ pela cultura e, consequentemente pouco tempo hábil para que as plantas apresentassem diferenças entre os tratamentos, assim como a grande interferência do solo em função da pequena biomassa
Tabela 1. Médias do índice de vegetação por diferença normalizada (NDVI) mensurado em diferentes épocase produtividade final de colmos nas parcelas dos tratamentos, com seus respectivos coeficientes de variação

\begin{tabular}{|c|c|c|c|c|c|c|c|c|}
\hline $\begin{array}{l}\text { Tratamento } \\
\left(\mathrm{kg} \mathrm{ha}^{-1} \text { de N) }\right.\end{array}$ & $\begin{array}{c}30 \text { DAC } \\
\text { NDVI }\end{array}$ & $\begin{array}{l}\text { CV } \\
(\%)\end{array}$ & $\begin{array}{c}60 \text { DAC } \\
\text { NDVI }\end{array}$ & $\begin{array}{l}\text { CV } \\
(\%)\end{array}$ & $\begin{array}{c}90 \text { DAC } \\
\text { NDVI }\end{array}$ & $\begin{array}{l}\text { CV } \\
(\%)\end{array}$ & $\begin{array}{l}\text { Produtividade } \\
\left(\mathrm{t} \mathrm{ha}^{-1}\right)\end{array}$ & $\begin{array}{l}\text { CV } \\
(\%)\end{array}$ \\
\hline 0 & 0,221 & 3,3 & 0,293 & 9,0 & $0,568 \mathrm{~b}$ & 6,0 & 59,3 & 6,6 \\
\hline 50 & 0,222 & 4,4 & 0,29 & 5,6 & $0,632 a$ & 5, & & 8,1 \\
\hline 100 & 0,223 & 4,9 & 0,300 & 7,6 & $0,636 a$ & 7,7 & & 6,3 \\
\hline 150 & 0,220 & 4,2 & 0,295 & 7,8 & $0,636 a$ & 5,8 & & 7,4 \\
\hline 200 & 0,220 & 4,5 & 0,301 & 7,7 & $0,660 \mathrm{a}$ & 4,5 & $72,8 \mathrm{a}$ & 7,6 \\
\hline Média & $0,221^{\text {ns }}$ & 4,3 & $0,297^{\mathrm{ns}}$ & 7,5 & $0,626^{*}$ & 5,8 & $67,0^{*}$ & 7,2 \\
\hline
\end{tabular}

DAC: dias após o corte; $\mathrm{CV}$ : coeficiente de variação; ${ }^{n s} \mathrm{e}$ *: respectivamente, não significativo $\mathrm{e}$ significativo ao nível de 0,05 de probabilidade; médias seguidas de letras iguais na coluna não diferem significativamente entre si, pelo teste de Tukey a $5 \%$

da cultura; observou-se, no entanto, aos 90 DAC efeito significativo nas mensurações de NDVI, tal como para a produtividade final de colmos.

$\mathrm{Na}$ avaliação aos 90 DAC o sensor foi capaz de diferenciar a resposta da cana-de-açúcar às doses de nitrogênio aplicadas, apresentando regressão linear com coeficiente de determinação de 0,7410. Esses resultados corroboram com os encontrados por Inamasu et al. (2006), ao testarem outro sensor de NDVI porém com bandas centradas em 590 e $880 \mathrm{~nm}$. Este tipo de constatação a partir de sensores ticos é possível em razão da grande absorção pela clorofila de ondas do espectro na região do vermelho e à forte refletância no infravermelho, em virtude da dispersão no mesófilo das folhas e da ausência de absorção pelos pigmentos (Woolley, 1971).

Observa-se que o aumento do NDVI mensurado sua amplitude reduzida conforme o aumento da dose de $\mathrm{N}$ aplicado sendo que estatisticamente apenas o tratamento que não recebeu $\mathrm{N}$ se diferenciou dos demais. Este fato se explica, segundo Argenta et al. (2001), porque em situações em que a disponibilidade de $\mathrm{N}$ é grande, o máximo potencial do sistema fotossintético se estabelece, sendo o excedente de $\mathrm{N}$ armazenado na forma de outros compostos de reserva.

Comportamento semelhante pode ser verificado pela produtividade final de colmos em resposta a diferentes doses de $\mathrm{N}$, onde a produtividade continuou aumentando conforme o incremento do $\mathrm{N}$ aplicado, apresentando um coeficiente de determinação de 0,9258 para regressão linear. Nas condições do presente estudo não foi possível se verificar queda na produtividade de colmos com a suposta aplicação excessiva $\operatorname{de} \mathrm{N}\left(200 \mathrm{~kg} \mathrm{ha}^{-1}\right)$.

Avaliando-se os modelos de regressão para NDVI e para produtividade de colmos verificou-se respectivamente um $\mathrm{R}^{2}$ de 0,74 e 0,93, sendo um bom indicativo da qualidade dos dados obtidos e da potencialidade da técnica. Kim et al. (2005), estudando dados de coleta com este mesmo sensor em três épocas diferentes e dois tipos de sistema de irrigação em cevada encontraram correlações entre o NDVI e a dose de nitrogênio aplicado na semeadura, de 63 a $90 \%$.

No gráfico da Figura 2 o NDVI mensurado aos 90 DAC se correlacionou positivamente com a produtividade final de colmos, obtidas nos diferentes tratamentos, fato que demonstra que se a cana-de-açúcar responder ao $\mathrm{N}$ em determinada situação o sensor terá capacidade de verificar esta resposta. 


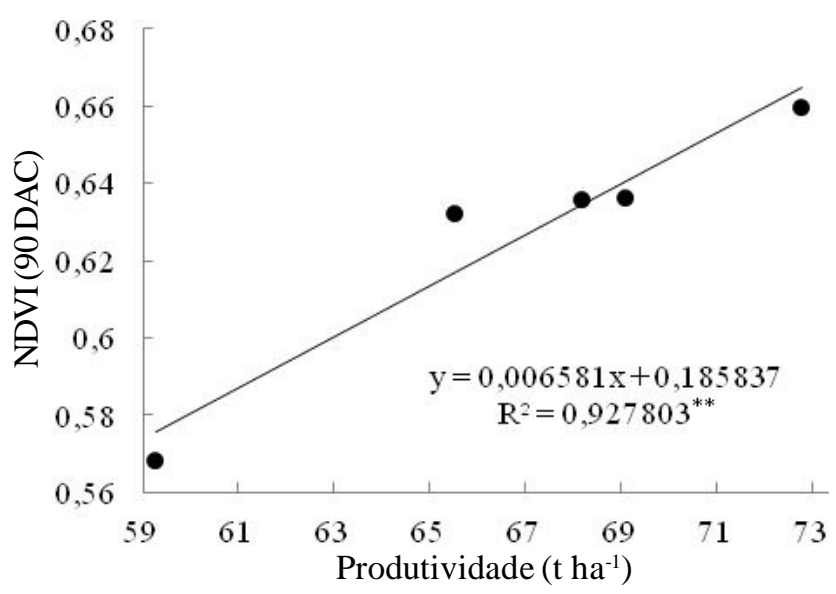

Figura 2. Correlação linear entre o NDVI mensurado aos 90 dias após colheita (DAC) e a produtividade final de colmos de cana-de-açúcar

Com estudos mais aprofundados e específicos para cada situação vislumbra-se a partir de algoritmos agronômicos de fertilização a possibilidade de utilização deste equipamento no auxílio à recomendação da adubação nitrogenada com aplicação em taxa variável e em tempo real. Seguem-se, assim, os preceitos da aplicação do fertilizante na dose e no local exatos de que a cultura necessita, proporcionando otimização na utilização de adubos nitrogenados e consequentes benefícios para o setor sucroalcooleiro e para o meio ambiente.

\section{CONClusÕES}

1. A cultura respondeu de forma linear às doses de nitrogênio aplicadas e o sensor ótico conseguiu captar esta resposta a partir de mensurações realizadas 90 dias após o corte da cana.

2. Este trabalho oferece subsídios para estudos mais aprofundados sobre o manejo em tempo real da adubação nitrogenada, por meio de sensores óticos e do NDVI.

3. O estudo indica que a técnica testada pode ser explorada visando à redução de custos de produção e impactos ambientais associados à adubação nitrogenada em cana-de-açúcar.

\section{AgRADECIMENTOS}

A FAPESP, Máquinas Agrícolas Jacto S.A., e ao Centro de Tecnologia Canavieira (CTC), que muito contribuíram para a realização deste trabalho.

\section{LITERATURA CITADA}

Argenta, G.; Silva, P. R. F.; Bortolini, C. G.; Forsthofer, E. L.; Strieder, M. L. Relação da leitura do clorofilômetro com os teores de clorofila extraível e de nitrogênio na folha de milho. Revista Brasileira de Fisiologia Vegetal, v.13, n.2, p.158-167, 2001.
Blackmer, T. M.; Schepers, J. S.; Varvel, G. E.; Walter-Shea, E. A. Nitrogen deficiency detection using reflected shortwave radiation from irrigated corn canopies. Agronomy Journal, v.88, p.1-5, 1996.

Grohs, D. S.; Bredemeier, C.; Mundstock, C. M.; Poletto, N. Modelo para estimativa do potencial produtivo em trigo e cevada por meio do sensor GreenSeeker. Engenharia Agrícola, v.29, n.1, p.101-112, 2009.

Inamasu, R. Y.; Sousa, R. V.; Porto, A. J. V.; Fortes, C.; Luchiari, A.; Schepers, J. S.; Shanahan, J. F.; Francis, D. D. Acesso ao estado nutricional da cana-de-açúcar por meio de sensor ativo de refletância. In: Congresso Brasileiro de Agricultura de Precisão, 2., 2006, São Pedro. Anais ... São Pedro: ESALQ/ USP, 2006. CD Rom

Kim, Y.; Evans, R. G.; Waddell, J. Evaluation of in-field optical sensor for nitrogen assessment of barley in two irrigation systems. ASAE paper No. PNW05-1004, St. Joseph: ASAE, 2005. 12p.

Min, M.; Lee, W. S. Determination of significant wavelengths and prediction on nitrogen content for citrus. Transactions of the ASAE, v.48, n.2, p.455-461, 2005.

Motomiya, A. V. A.; Molin, J. P.; Chiavegato, E. J. Utilização de sensor óptico ativo para detectar deficiência foliar de nitrogênio em algodoeiro. Revista Brasileira de Engenharia Agrícola e Ambiental, v.13, n.2, p.137-145, 2009.

Piekielek, W. P.; Foz, R. H.; Toth, J. D.; Macneal, K. E. Use of a chlorophyll meter at the early dent stage of corn to evaluate nitrogen sufficiency. Agronomy Journal, v.87, p.403-408, 1995.

Povh, F. P.; Molin, J. P.; Gimenez, L. M.; Pauletti, V.; Molin, R.; Salvi, J. V. Comportamento do NDVI obtido por sensor ótico ativo em cereais. Pesquisa Agropecuária Brasileira, v.23, n.8, p.1075-1083, 2008.

Prado, H. Ambientes de produção de cana-de-açúcar na região Centro-Sul do Brasil. Informações Agronômicas, v.110, p.1217, 2005. Encarte Técnico

Raun, W. R.; Solie, J. B.; Johnson, G. V.; Stone, M. L.; Mullen, R. W.; Freeman, K. W.; Thomason, W. E.; Lukina, E. V. Improving nitrogen use eficiency in cereal grain production with optical sensing and variable rate application. Agronomy Journal, v.94, p.815-820, 2002.

Segato, S. V.; Pinto, A. S.; Jendiroba, E.; Nóbrega, J. C. M. Atualização em produção em cana-de-açúcar. Piracicaba: EdLivroceres, 2006. 415p.

Stone, M. L.; Solie, J. B.; Raun, W. R.; Whitney, R. W.; Taylor, S. L.; Ringer, J. D. Use of spectral radiance for correcting inseason fertilizer nitrogen deficiencies in winter wheat. Transactions of the ASAE, v.39, n.5, p.1623-1631, 1996.

Sui, R.; Wilkerson, J. B.; Hart, W. E.; Wilhelm, L. R., Howard, D. D. Multi-spectral sensor for detection of nitrogen status in cotton. Applied Engineering in Agriculture, v.21, n.2, p.167172, 2005.

Tarpley, L.; Reddy, K. R.; Sassenrath-Cole, G. F. Reflectance indices with precision and accuracy in predicting cotton leaf nitrogen concentration. Crop Science, v.1, n.40, p.18141819, 2000. 
Teal, R. K.; Tubana, B.; Girma, K.; Freeman, K. W.; Arnall, D. B.; Walsh, O.; Raun, W. R. In-season prediction of corn grain yield potential using normalized difference vegetation index. Agronomy Journal, v.98, p.1488-1494, 2006.

UDOB - União dos Produtores de Bioenergia. Programa de melhoramento genético da cana-de-açúcar. http://www.udop.com.br/ index.php?item=variedades_rb. 16Jun. 2009.
Woolley, J. T. Reflectance and transmittance of light by leaves. Plant Physiology, v.47, p.656-662, 1971.

Wright, D. L.; Rasmussen, V. P.; Ransey, R. D.; Baker, D. J.; Ellsworth, J. W. Canopy reflectance estimation of wheat nitrogen content for grain protein management. GIScience and Remote Sensing, v.41, n.4, p.287-300, 2004. 